\title{
Cuidado farmacêutico em utentes idosos portadores de asma atendidos na farmácia ambulatorial do Hospital Universitário João de Barros Barreto, Belém, Pará
}

Pharmaceutical care for elderly asthma patients attended at the outpatient pharmacy of João de Barros Barros Barreto, Belém, Pará

Atención farmacéutica a utentes ancianos con asma atendidos en la farmacia de utentes ambulatorios de João de Barros Barros Barreto, Belém, Pará

\author{
Maike Wendel Paes \\ ORCID: https://orcid.org/0000-0003-2760-0906 \\ Universidade Federal do Pará, Brasil \\ E-mail: maikepaes03@gmail.com \\ Alan Barroso Araújo Grisólia \\ ORCID: https://orcid.org/0000-0002-8004-0277 \\ Universidade Federal do Pará, Brasil \\ E-mail: alangrisolia@gmail.com \\ Orenzio Soler \\ ORCID: https://orcid.org/0000-0003-2246-0019 \\ Universidade Federal do Pará, Brasil \\ E-mail: orenziosoler@gmail.com
}

\begin{abstract}
Resumo
Objetivo: Proporcionar o cuidado farmacêutico por meio de serviços farmacêuticos voltados aos utentes idosos portadores de asma atendidos em farmácia ambulatorial. Método: Estudo de caráter intervencional, transversal e prospectivo, realizado no período de julho de 2019 a dezembro de 2019. Durante as consultas farmacêuticas coletaramse dados farmacoterapêuticos, identificou-se os problemas relacionados a medicamentos e realizaram-se serviços farmacêuticos por meio de intervenções organizacionais e educacionais. Resultados: 26 utentes finalizaram o acompanhamento, a maioria do sexo feminino (84,62\%), residentes em Belém $(84,62 \%)$, escolaridade ensino médio (46,15\%), ocupação aposentado (69,23\%). Mais da metade $(53,85 \%)$ estava com sobrepeso, $(61,53 \%)$ não praticava atividade física. As comorbidades mais prevalentes foram hipertensão $(65,41 \%)$ e dislipidemias $(42,36 \%)$. Os Problemas Relacionados aos Medicamentos mais prevalentes foram o problema de saúde insuficientemente tratado (20,28\%), e medicamentos administrados incorretamente (17,39\%). Após as diferentes intervenções observou-se melhora da adesão ao tratamento medicamentoso $(\mathrm{p}<0,01)$, maior controle da doença $(\mathrm{p}<0,01)$, melhor função pulmonar $(\mathrm{p}<0,01)$ e melhoria na qualidade de vida $(\mathrm{p}<0,01)$. Conclusão: A prática do cuidado farmacêutico realizado por meio de serviços farmacêuticos diretamente destinados aos utentes idosos com asma melhorou significativamente os desfechos clínicos e humanísticos nessa população.
\end{abstract}

Palavras-chave: Idosos; Asma; Farmácia; Cuidado farmacêutico; Intervenções farmacêuticas.

\begin{abstract}
Objective: To provide pharmaceutical care through pharmaceutical services aimed at elderly patients with asthma treated in an outpatient pharmacy. Method: Interventional, cross-sectional and prospective study, carried out from July 2019 to December 2019. During pharmaceutical consultations, pharmacotherapeutic data were collected, problems related to medications were identified and pharmaceutical services were performed through organizational and educational interventions. Results: 26 users completed the follow-up, most of them female (84.62\%), residing in Belém $(84.62 \%)$, high school education $(46.15 \%)$, retired occupation $(69.23 \%)$. More than half $(53.85 \%)$ were overweight, $(61.53 \%)$ did not practice physical activity. The most prevalent comorbidities were hypertension $(65.41 \%)$ and dyslipidemia (42.36\%). The most prevalent Drug Related Problems were poorly treated health problem (20.28\%), and incorrectly administered drugs (17.39\%). After the different interventions, there was an improvement in adherence to drug treatment $(\mathrm{p}<0.01)$, greater disease control $(\mathrm{p}<0.01)$, better lung function $(\mathrm{p}<0.01)$ and improvement in quality of life $(\mathrm{p}<0.01)$. Conclusion: The practice of pharmaceutical care carried out through pharmaceutical services directly aimed at elderly patients with asthma significantly improved clinical and humanistic outcomes in this population.
\end{abstract}

Keywords: Elderly; Asthma; Pharmacy; Pharmaceutical care; Pharmaceutical interventions. 


\begin{abstract}
Resumen
Objetivo: Brindar atención farmacéutica a través de servicios farmacéuticos dirigidos a pacientes ancianos con asma atendidos en una farmacia ambulatoria. Método: Estudio intervencionista, transversal y prospectivo, realizado de julio de 2019 a diciembre de 2019. Durante las consultas farmacéuticas se recolectaron datos farmacoterapéuticos, se identificaron problemas relacionados con medicamentos y se realizaron servicios farmacéuticos a través de intervenciones organizativas y educativas. Resultados: 26 usuarios completaron el seguimiento, la mayoría mujeres (84,62\%), residentes en Belém (84,62\%), educación secundaria (46,15\%), ocupación jubilada (69,23\%). Más de la mitad $(53,85 \%)$ tenían sobrepeso, $(61,53 \%)$ no practicaba actividad física. Las comorbilidades más prevalentes fueron hipertensión $(65,41 \%)$ y dislipidemia $(42,36 \%)$. Los problemas relacionados con las drogas más prevalentes fueron problemas de salud mal tratados $(20,28 \%)$ y medicamentos administrados incorrectamente $(17,39 \%)$. Tras las diferentes intervenciones, hubo una mejora en la adherencia al tratamiento farmacológico $(\mathrm{p}<0,01)$, mayor control de la enfermedad ( $\mathrm{p}<0,01)$, mejor función pulmonar $(\mathrm{p}<0,01)$ y mejoría en la calidad de vida ( $\mathrm{p}<0,01)$. Conclusión: La práctica de la atención farmacéutica realizada a través de servicios farmacéuticos dirigidos directamente a pacientes ancianos con asma mejoró significativamente los resultados clínicos y humanísticos en esta población.
\end{abstract}

Palabras clave: Anciano; Asma; Farmacia; Cuidado farmacéutico; Intervenciones farmacéuticas.

\title{
1. Introdução
}

Asma é uma doença crônica caracterizada por inflamação das vias aéreas, na qual muitas células e elementos celulares têm participação. A inflamação crônica está associada à hiperresponsividade das vias aéreas; assim provocando limitação variável do fluxo expiratório, aumento na produção de muco e espessamento da parede brônquica. Entre os sintomas mais característicos estão dispneia, sibilos, opressão torácica e tosse; principalmente à noite ou no início da manhã. Esses episódios são uma consequência da obstrução ao fluxo aéreo intrapulmonar generalizado e variável, reversível espontaneamente ou com tratamento (Global Initiative for Asthma, 2019; National Institutes of Health, 2018; Boulet et al., 2019).

A prevalência global da asma situa-se entre 6 e $7 \%$ na população em geral e estima-se ser 7 a $9 \%$ nos idosos. Os idosos não só sofrem de um fardo desproporcional da doença, quando comparados com outros grupos, como de maior risco de morrerem de asma, apresentando uma maior taxa de mortalidade (51,1/1000000 habitantes) do que qualquer outro grupo etário. Estima-se que atualmente existem 235 milhões de pessoas com asma no mundo. Estando presente em todos os países, independentemente do seu nível de desenvolvimento e que mais de $80 \%$ das mortes por asma ocorrem em países de baixa e média-baixa renda. No Brasil, segundo a Pesquisa Nacional de Saúde do Ministério da Saúde e do Instituto Brasileiro de Geografia e Estatística (IBGE), 6,4 milhões de brasileiros com mais de 18 anos têm asma, doença crônica mais comum no país que afeta as vias respiratórias e o pulmão. Considerando população adulta, crianças e adolescentes, pode chegar a aproximadamente 20 milhões de pessoas (Instituto Brasileiro de Geografia e Estatística, 2013; Global Initiative for Asthma, 2019; National Institutes of Health, 2018; Boulet et al., 2019).

O tratamento, de acordo com a Diretriz Brasileira para o Manejo da Asma, deve ser realizado de acordo com a classificação da asma, abordando aspectos como a farmacoterapia anti-inflamatória inalatória ou sistêmica, broncodilatadores $\beta 2$-agonistas de curta ou longa duração, associados ao controle dos fatores desencadeantes domiciliares e ocupacionais. Isso implica em uma abordagem personalizada, incluindo tratamento farmacológico, educação do paciente, plano de ação por escrito, treinamento para uso do dispositivo inalatório e revisão da técnica inalatória a cada visita ao consultório (Paes et al., 2020; Pizzichini, 2020).

A via preferencial de tratamento farmacológico é a via inalatória, dado seus efeitos diretos na via respiratória e o menor risco de efeitos adversos sistêmicos. Contudo, os benefícios desta via de tratamento são altamente dependentes de uma técnica inalatória correta aplicada pelo paciente (Global Initiative for Asthma, 2019). De acordo com as evidências, apenas a metade dos asmáticos utiliza corretamente o medicamento prescrito (Chipps et al., 2020), especialmente os idosos que apresentam grandes dificuldades em realizar a técnica inalatória de forma correta, comumente utilizando esses dispositivos de maneira subótima, o que pode conduzir à maior procura por atendimentos de emergência e hospitalizações evitáveis (Janežič et al., 2019). 
Quando se trata de idosos portadores de asma, outro fator que pode interferir no controle da doença é a utilização de múltiplos fármacos que pode ser necessária em muitas ocasiões. Assim, sendo, a polifarmacoterapia deve ser adequadamente supervisionada; uma vez que aumenta o risco de interações medicamentosas, efeitos adversos e redundância terapêutica, podendo resultar em iatrogenias, internações e gastos desnecessários (Nastaravicius \& Ramanauskiene, 2018).

Neste contexto, o profissional farmacêutico é figura primordial para o adequado tratamento farmacológico e para o controle da doença. Ao realizar intervenções farmacêuticas por meio da prática conhecida como cuidado farmacêutico visando uma farmacoterapia racional - o que se dá pela interação direta do farmacêutico com o utente -, obtem-se resultados definidos e mensuráveis voltados para a melhoria da qualidade de vida (Nastaravicius \& Ramanauskiene, 2018; Paes et al., 2020; Pizzichini et al., 2020).

Estudos têm reforçado a ideia de que intervenções farmacêuticas aumenta a adesão dos utentes idosos a seus regimes terapêuticos, podendo promover redução de custos hospitalares; ou seja, diminuição do número de prescrições, de internações e de medicamentos associados a reações adversas a medicamentos (Nastaravicius \& Ramanauskiene, 2018; Paes et al., 2020; Pizzichini et al., 2020). Nesta perspectiva, este estudo buscou proporcionar o cuidado farmacêutico em utentes idosos portadores de asma atendidos na Farmácia Ambulatorial do Hospital Universitário João de Barros Barreto da Universidade Federal do Pará, visando o uso com qualidade de medicamentos e melhor efetividade do tratamento.

\section{Metodologia}

\section{Desenho da pesquisa}

Trata-se de uma pesquisa-ação, do tipo transversal, prospectiva, analítica e descritiva (Minayo, 2018; Thiollent, 2011; Yin, 2015), por amostragem de conveniência, utilizando-se de intervenções farmacêuticas coordenadas e aplicadas a um grupo de idosos portadores de asma atendidos na Farmácia Ambulatorial no período de julho de 2019 a dezembro de 2019.

\section{Local do estudo}

O Serviço de Farmácia Ambulatorial do Hospital Universitário João de Barros Barreto (HUJBB) da Universidade Federal do Pará (UFPA), em Belém do Pará, realiza atendimento aos utentes com asma, proporcionando a dispensação de medicamentos do Componente Especializado da Assistência Farmacêutica (CEAF) que se dá através da Autorização de Procedimento de Alta Complexidade/Custo (APAC) pelo Ministério da Saúde, por meio do sistema informatizado HÓRUSEspecializado. Em 2019 a Farmácia Ambulatorial possui 582 utentes portadores de asma com cadastro ativo, deste, 317 (54,46\%) eram idosos. O serviço de Farmácia Ambulatorial dispõe de quatro farmacêuticos, os quais dispensam mensalmente os medicamentos regulamentados pela portaria GM/MS nº 2.981 de 26 de novembro de 2009.

\section{População do estudo}

Foram convidados 50 utentes com diagnóstico de asma que frequentavam regularmente a farmácia para recebimento de seus medicamentos (formoterol + budesonida). 40 utentes compareceram a primeira consulta; sendo que 26 finalizaram o acompanhamento farmacoterapêutico após seis meses.

\section{Critérios de inclusão e exclusão}

Foram incluídos no estudo os utentes com idade igual ou superior a 60 anos, com diagnóstico clínico de asma e em tratamento com broncodilatador associado a um corticoide (formoterol + budesonida), cadastrados na Farmácia Ambulatorial do HUJBB, a qual dispensa medicamentos do Componente Especializado da Assistência Farmacêutica, com condições de responder aos questionários, e que concordaram em assinar o Termo de Consentimento Livre e Esclarecido (TCLE). Foram inelegíveis os 
utentes fumantes, os que não concordaram e não assinaram o Termo de Consentimento Livre e Esclarecido (TCLE); bem como, aqueles que não apresentaram condições cognitivas e motoras para responder ao questionário.

\section{Aspectos éticos}

Os dados do estudo foram obtidos após a aprovação do projeto de pesquisa pelo Comitê de Ética e Pesquisa do Hospital Universitário João de Barros Barreto (HUJBB), sob o CAAE: 10331419.8.0000.0018 e Parecer Consubstanciado nº 3.404.978, respeitando os preceitos da Resolução do Conselho Nacional de Saúde no 466 de 12 de dezembro de 2012 e da Resolução ${ }^{\circ} 510$, de 07 de abril de 2016, que regulamentam os estudos científicos envolvendo seres humanos (Brasil, 2012; 2016). Para início do acompanhamento farmacêutico fez-se necessário o preenchimento do Termo de Consentimento Livre e Esclarecido (TCLE). Manteve-se sigilo dos dados de identificação e informações pertinentes aos participantes da pesquisa.

\section{Coleta e análise dos dados}

Os dados foram coletados por um único farmacêutico durante as consultas farmacêuticas, em um consultório reservado. Para a coleta de dados fez uso de um formulário de acompanhamento farmacêutico adaptado de Brasil (2014) e de Dantas et al. (2019), onde se fez o registro de variáveis sociodemográficas como idade, sexo, renda, escolaridade; assim como, das informações relacionadas à farmacoterapia, como medicamentos utilizados, dificuldades enfrentadas durante o tratamento e outras questões relativas à doença.

Durante os seis meses de acompanhamento farmacêutico ocorreram encontros mensais com cada utente, sendo que no primeiro contato fez-se a apresentação do estudo, aplicação do Termo de Consentimento Livre e Esclarecido (TCLE), dando-se início as consultas farmacêuticas para potenciais intervenções farmacêuticas, seguindo o modelo preconizado por Correr e Otuki (2013) (Figura 1).

Figura 1 - Método clínico do cuidado farmacêutico ao paciente.

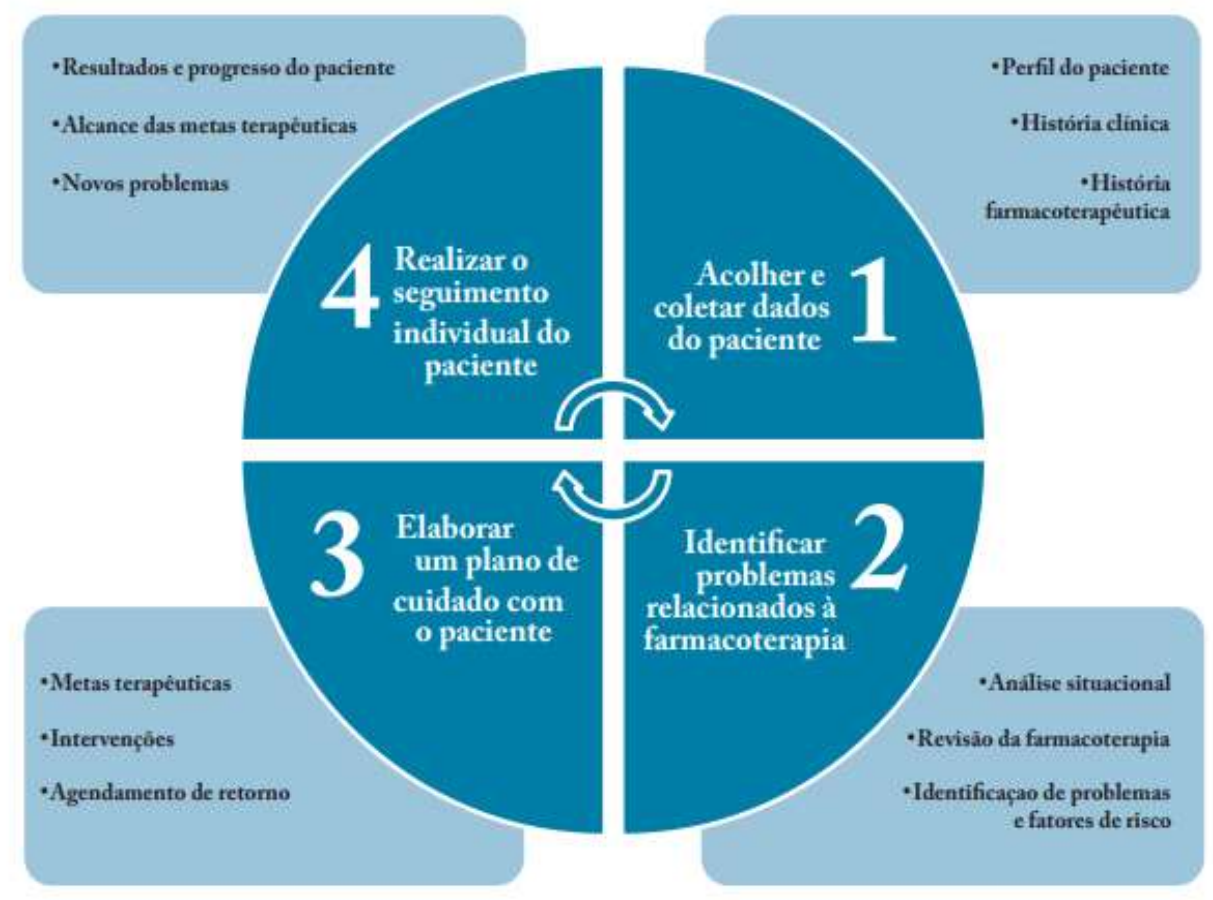

Fonte: Reproduzido de Correr e Otuki (2013). 


\section{Avaliação de desfechos clínicos e humanísticos}

Optou-se por realizar avaliações mensais dos níveis de controle da asma, do fluxo expiratório máximo; assim como, na primeira e na última sessão, identificou-se o grau de adesão á farmacoterapia e o escore de qualidade de vida dos utentes, utilizando-se instrumentos específicos.

\section{Avaliação do pico de fluxo expiratório}

Em todos os encontros os utentes passaram por avaliação do pico de fluxo expiratório; que é a velocidade máxima alcançada pelo ar na expiração forçada, curta e rápida, após máxima inspiração. Para medir o pico do fluxo expiratório foi utilizado o aparelho peak flow. O peak flow é de funcionamento é simples, onde o fluxo de ar expirado move uma mola calibrada, que arrasta um medidor até alcançar o pico de fluxo máximo, ponto em que se fará a leitura do resultado do teste na escala. Posteriormente a mole é reconduzida à posição normal, podendo o aparelho ser usado para novas medidas após a troca do bocal descartável (Gardenghi et al., 2010; Fuller et al., 2017; Yadav \& Thapa, 2019).

O peak flow é pequeno, portátil e vem ganhando espaços crescentes, possibilitando uma medida de função pulmonar não somente em laboratórios, mas também no âmbito hospitalar, ambulatorial e até mesmo domiciliar. Sua importância reside no fato de ser um método confiável, simples e de baixo custo. O resultado da medida proporcionada por este aparelho contribui para o diagnóstico da asma; bem como, para o monitoramento, possibilitando avaliar a evolução da obstrução do fluxo aéreo e da resposta terapêutica (Aguiar et al., 2017; Almomani et al., 2018).

Para realização da técnica solicitou-se que os utentes se mantivessem sentados, e realizassem uma inspiração máxima seguida de uma expiração forçada máxima. Esse procedimento se repetiu por três vezes, com intervalo de um minuto entre cada um deles, registrando, dos três valores medidos, o maior valor obtido (Dalcin et al, 2014; Aguiar et al., 2017; Almomani et al., 2018).

\section{Avaliação do nível de controle da asma}

Para o monitoramento do controle da asma, os utentes em acompanhamento também realizaram em todos os encontros o Asthma Control Test (ACT), que foi desenvolvido e validado por Nathan et al., (2004). O ACT foi desenvolvido para avaliar o controle da asma e tem mostrado fortes propriedades avaliativas e discriminativas. Consiste em cinco questões, relacionadas às quatro semanas que antecedem a avaliação, abordando múltiplas dimensões de controle, incluindo episódios de falta de ar, despertares noturnos, limitações nas atividades de vida diária, autoavaliação de controle da asma e necessidade de medicação de alívio. O escore total varia de 5 a 25 pontos. Um agrupamento desta pontuação permite definir três graus de controle da asma nas últimas 4 semanas, da seguinte forma: <20: "Asma não controlada"; 20 -24: "Asma parcialmente controlada"; 25: "Asma completamente controlada". ACT foi traduzido para o português e mostrou-se um questionário válido, reprodutível e sensível para a avaliação do controle da asma em uma amostra de utentes ambulatoriais no Brasil, com baixa escolaridade e atendida em um hospital terciário do Sistema Único de Saúde (Roxo et al., 2010; Brasil, 2013; Oliveira et al., 2014; Aguiar et al., 2017; Almomani et al., 2018).

\section{Identificação da adesão a farmacoterapia}

A ferramenta de escolha para identificação da adesão a farmacoterapia foi o Brief Medication Questionnaire (BMQ). Instrumento de investigação da adesão composto por três domínios com perguntas que identificam possíveis barreiras à adesão quanto ao regime, às crenças e à recordação em relação ao tratamento medicamentoso na perspectiva do utente. Foi utilizada a versão do BMQ traduzida para o português e submetida a estudo de validação que classifica a adesão de acordo com um escore composto com o número de respostas positivas em alta adesão e/ou nenhuma adesão: provável alta adesão (1), provável baixa 
adesão (2) e baixa adesão (3 ou mais) em qualquer domínio. Sendo esta avaliação realizada em dois momentos, no primeiro contato com os utentes; ou seja, sem a provisão do cuidado e no último encontro ao final do acompanhamento farmacoterapêutico. Neste estudo foram considerados com baixa adesão apenas aqueles que obtiveram três ou mais respostas positivas em qualquer domínio (Leite et al., 2008; Bem et al., 2012; Dantas et al., 2018).

\section{Determinação da qualidade de vida}

O instrumento World Health Organization Quality of Life Group Old (WHOQOL-OLD) avalia e reconhece áreas específicas de qualidade de vida que podem ser mais importantes para os idosos (Power et al., 2005). Composto por seis facetas (ou domínios) de quatro itens cada (o módulo consiste em 24 itens) avaliados pela da escala Likert (1 a 5 pontos) atribuídos a seis facetas. Para todos os domínios, o escore dos valores possíveis pode oscilar de 4 a 20; desde que todos os itens dos domínios tenham sido preenchidos. Os escores dos seis domínios ou os valores dos 24 itens do módulo WHOQOL-OLD podem ser combinados para produzir um escore geral para a qualidade de vida em idosos, denotado como o "escore total". Assim, sendo, escores mais altos representam uma alta qualidade de vida e escores baixos representam uma baixa qualidade de vida (Flecka et al., 2003; Power \& Quinn, 2005; Cavalcante et al., 2018). A qualidade de vida foi avaliada antes do fornecimento do cuidado farmacêutico e ao final do período de acompanhamento farmacoterapêutico.

\section{Análise estatística}

Para realização das análises estatísticas os dados foram compilados em planilhas do Microsoft Exce $^{\circledR} 2018$. Posteriormente foram analisados utilizando o software estatístico GraphPadPrism (Versão 8.0). Os dados referentes as variáveis sociodemográficas, antropométricas, comorbidades e dados farmacoterapêuticos foram representados em figuras e tabelas e tratados a partir da estatística descritiva.

O teste de Shapiro-Wilk foi aplicado para verificar a normalidade dos dados. Depois de confirmada a distribuição anormal dos dados, o teste não-paramétrico de Wilcoxon para amostras pareadas foi utilizado para comparação intragrupo inicial e final das variáveis de desfechos clínicos e humanísticos (controle da asma, fluxo expiratório, adesão a farmacoterapia e qualidade de vida). Para todos os procedimentos, adotou- se um intervalo de confiança de 5\%. Foi considerada diferença significativa $p$ valor menor que $0,05(\mathrm{p}<0,05)$.

\section{Resultados e discussão}

\section{Perfil sociodemográfico dos utentes}

Observa-se, que dos 26 utentes acompanhado, 22 (84,62\%) são do sexo feminino, 13 (50\%) estão na faixa etária entre 60 e 69 anos, 19 (73,07\%) se autodeclararam pardos ou morenos, 12 (46,15\%) relataram ter ensino médio, 18 (69,23\%) são aposentados, $10(38,47 \%)$ são solteiros, 26 (100\%) tem uma renda familiar mensal entre 1 e 3 salários-mínimos e $22(84,62 \%)$ residem em Belém, Pará (Tabela 1). 
Tabela 1 - Perfil sociodemográfico.

\begin{tabular}{|c|c|c|}
\hline Variável & $\begin{array}{l}\text { Frequência Absoluta } \\
(\mathbf{N})\end{array}$ & $\begin{array}{c}\text { Frequência Relativa } \\
\%\end{array}$ \\
\hline \multicolumn{3}{|c|}{ Perfil Sociodemográfico } \\
\hline \multicolumn{3}{|l|}{ Sexo } \\
\hline F & 22 & 84,62 \\
\hline M & 4 & 15,38 \\
\hline \multicolumn{3}{|l|}{ Idade } \\
\hline $60-69$ & 13 & 50 \\
\hline $70-79$ & 12 & 46,15 \\
\hline$\geq 80$ & 1 & 3,85 \\
\hline \multicolumn{3}{|l|}{ Cidade } \\
\hline Belém & 22 & 84,62 \\
\hline Ananindeua & 4 & 15,38 \\
\hline \multicolumn{3}{|l|}{ Raça ou cor da pele } \\
\hline Parda ou Morena & 19 & 73,07 \\
\hline Branca & 4 & 15,38 \\
\hline Preta & 2 & 7,7 \\
\hline Amarela & 1 & 3,85 \\
\hline \multicolumn{3}{|l|}{ Escolaridade } \\
\hline Ensino Superior & 2 & 7,7 \\
\hline Ensino Médio & 12 & 46,15 \\
\hline Ensino Fundamental & 11 & 42,31 \\
\hline Analfabeto & 1 & 3,85 \\
\hline \multicolumn{3}{|l|}{ Estado Civil } \\
\hline Casado & 8 & 30,77 \\
\hline Solteiro & 10 & 38,47 \\
\hline Separa/Divorciado & 4 & 15,38 \\
\hline Viúvo & 4 & 15,38 \\
\hline \multicolumn{3}{|l|}{ Renda Familiar mensal } \\
\hline 1 a $3 \mathrm{SM}$ & 26 & 100 \\
\hline \multicolumn{3}{|l|}{ Habitantes no domicílio } \\
\hline 1 a 3 & 21 & 80,77 \\
\hline 4 a 7 & 5 & 19,23 \\
\hline \multicolumn{3}{|l|}{ Ocupação } \\
\hline Aposentado & 18 & 69,23 \\
\hline Desempregado & 5 & 19,22 \\
\hline Funcionário Público & 1 & 3,85 \\
\hline Profissional liberal & 1 & 3,85 \\
\hline Pensionista & 1 & 3,85 \\
\hline \multicolumn{3}{|l|}{ Religião } \\
\hline Catolicismo & 20 & 76,92 \\
\hline Protestante & 6 & 23,08 \\
\hline
\end{tabular}

Nota: $\mathrm{n}=26$. Fonte: Cuidado farmacêutico em utentes idosos portadores de asma atendidos na farmácia ambulatorial do hospital universitário João de Barros Barreto, Belém, Pará.

Quanto ao estado nutricional, 14 (53,85\%) apresentou-se com sobrepeso e, quando perguntados sobre os hábitos de vida, $16(61,53 \%)$ relataram não praticar atualmente nenhuma atividade física, 20 (76,92\%) não ingerem bebidas alcoólicas e 16 $(61,53 \%)$ informaram ser ex-fumantes (Tabela 2). 
Tabela 2 - Estado nutricional e hábitos de vida.

\begin{tabular}{|c|c|c|}
\hline Variável & $\mathbf{N}$ & $\%$ \\
\hline \multicolumn{3}{|c|}{ Antropometria e Hábitos de vida } \\
\hline \multicolumn{3}{|l|}{ Estado Nutricional } \\
\hline Baixo peso & 3 & 11,54 \\
\hline Adequado ou eutrófico & 9 & 34,61 \\
\hline Sobrepeso & 14 & $\mathbf{5 3 , 8 5}$ \\
\hline \multicolumn{3}{|l|}{ Prática de atividades físicas } \\
\hline Pratica Atividades & 10 & 38,47 \\
\hline Não Pratica Atividades & 16 & 61,53 \\
\hline \multicolumn{3}{|l|}{ Hábito de fumar } \\
\hline Nunca fumou & 10 & 38,47 \\
\hline Ex-fumante & 16 & $\mathbf{6 1 , 5 3}$ \\
\hline \multicolumn{3}{|l|}{ Consumo de álcool } \\
\hline Não ingere & 20 & 76,92 \\
\hline Ingere moderadamente & 6 & 23,08 \\
\hline \multicolumn{3}{|c|}{$\begin{array}{l}\text { Nota: } \mathrm{n}=26 \text {. Fonte: Cuidado farmacêutico } \\
\text { em utentes idosos portadores de asma } \\
\text { atendidos na farmácia ambulatorial do } \\
\text { hospital universitário João de Barros } \\
\text { Barreto, Belém, Pará. }\end{array}$} \\
\hline
\end{tabular}

Reconhece-se, que nesse grupo de utentes - os idosos - a falta de integração e centralização do cuidado tem como consequência o atendimento por diversas especialidades médicas e uso de múltiplos medicamentos. Há evidências de que essa população é a mais medicalizada na sociedade, representando cerca de 50\% dos consumidores de medicamentos no Brasil, com prevalência média do uso de 5 fármacos/dia; podendo chegar a 14 fármacos/dia. Assim, sendo, há necessidade de intervenções farmacêuticas direcionadas para o uso racional de medicamentos (Anum et al., 2017; Kovacevic et al., 2018; Soler \& Barreto, 2019). Em adição, Ferreira e Soler, (2020) apresentam evidências de que existem distintas estratégias empregadas para promover a segurança no uso de medicamentos e o farmacêutico - como consultor técnico e clínico sobre medicamentos -, pode atuar detectando potenciais falhas e traçando estratégias para promover a segurança do paciente com vistas a reduzir custos com tecnologias da saúde. Outrossim, há também, distintas intervenções que promovem práticas seguras do uso de medicamentos no ambiente hospitalar (Oliveira et al., 2021).

Identificou-se que o sexo feminino se fez mais prevalente $(84,62 \%)$, confirmando os dados obtidos na última Pesquisa Nacional de Saúde realizada pelo Instituto Brasileiro de Geografia e Estatística (IBGE), que identificou as mulheres como as mais acometidas pela asma no Brasil com prevalência de 39\% a mais entre o sexo feminino (Instituto Brasileiro de Geografia e Estatística, 2019). Esse achado também pode ser explicado devido ao aumento da expectativa de vida da população brasileira, que tem acontecido especialmente entre as mulheres, o que pode influir na presença de maioria feminina nesse estudo e que corrobora com outros realizados no país (Andrade et al., 2015).

Sobre a renda familiar mensal, identificou-se integralidade da população com renda entre um e três salários-mínimos, caracterizando a amostra como de classe baixa segundo critérios do Instituto Brasileiro de Geografia e Estatística (IBGE) na pesquisa de orçamentos familiares (Instituto Brasileiro de Geografia e Estatística, 2019). Isso reforça a necessidade de maior atenção a este grupo, quando se trata da prestação de cuidados a saúde, pois o Brasil está entre os países com maiores graus de desigualdade socioeconômica. Estudos tem demonstrado a existência de associações entre menor renda domiciliar per capita entre idosos e pior condição de saúde, pior função física, assim como maior dificuldade de acesso aos serviços de saúde, mesmo quando a cobertura desses serviços é adequada (Costa et al., 2019). 
Observou-se, predomínio de idosos que se encontravam com sobrepeso e que praticam nenhuma atividade física. Estudos nacionais e internacionais vêm demostrando que o excesso de peso está associado a maior manifestação de sintomas e piora da gravidade da asma (Cardoso et al., 2017; Costa et al., 2019). Reconhece-se que utentes com asma moderada e grave apresentam melhora da capacidade aeróbica e da qualidade de vida, além de redução no aparecimento de sintomas em utentes submetidos a um programa de condicionamento físico (Lopes et al., 2013; Poudel et al., 2015; Anum et al., 2017; Amegadzie et al., 2019).

Quanto ao consumo de álcool e tabaco, sabe-se que o tabagismo exacerba a asma nos adultos, restringindo a sua atividade, contribuindo para suas deficiências e aumentando o risco de asma grave, incrementando a necessidade de atendimentos de urgências (Word Health Organization, 2019). Nenhum dos idosos relatarem tabagismo atual, sendo que mais de 60\% relataram passado tabagista, o que deve ser tratado como sinal de alerta para possíveis crises. Há registros de que a proporção de asmáticos graves com passado de tabagismo é maior (Pinheiro et al., 2018). Mais de 20\% dos utentes com asma relatou ser consumidor de bebidas alcoólicas. O álcool pode provocar resposta imunitária o que pode refletir no controle da asma alérgica, pois acreditase que o consumo de álcool suprime a resposta imunológica dependente de Th1 específica a alérgenos e distorce a resposta Th2, o que leva a aumento da produção e liberação de citocinas e de imunoglobulina E (IgE). Logo, indivíduos com asma, sobretudo com alto consumo de álcool, necessitam de intervenções educacionais direcionadas a esse hábito, pois apresentam maior risco de apresentaram doenças respiratórias alérgicas (Linneberg \& Gonzalez, 2016).

\section{Perfil farmacoterapêutico e morbidades}

$20(76,92 \%)$ utentes apresentava mais de um problema de saúde diagnosticado. $14(53,84 \%)$ apresentaram entre três a quatro diagnósticos diferentes, estando entres as comorbidades mais prevalentes a asma (100\%), seguidas por hipertensão $(65,41 \%)$, dislipidemias $(42,36 \%)$ e rinite $(15,47 \%)$ (Figura 2$)$.

Figura 2 - Distribuição das comorbidades da população do estudo.

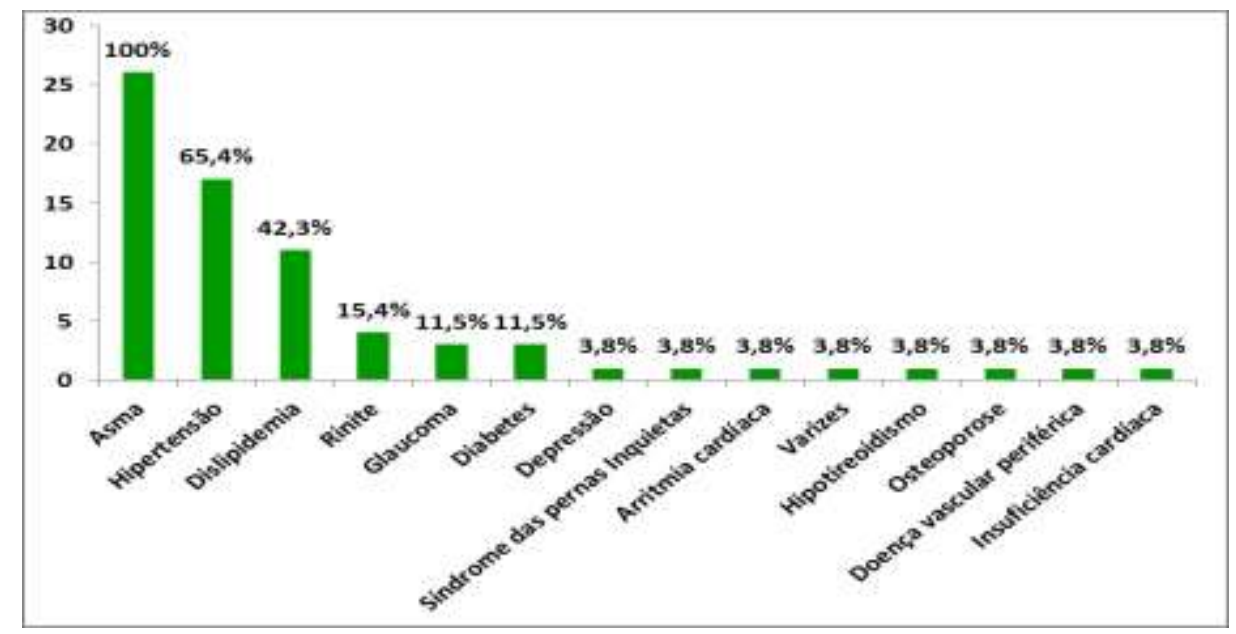

Nota: $\mathrm{n}=26$. Fonte: Cuidado farmacêutico em utentes idosos portadores de asma atendidos na farmácia ambulatorial do hospital universitário João de Barros Barreto, Belém, Pará.

A média de medicamentos (fármacos) utilizados por paciente foi de 5,6. 11 (42,31\%) utentes utilizavam 7 ou mais fármacos concomitantes. Identificou-se que os medicamentos mais utilizados foram para o sistema cardiovascular (60), sistema respiratório (55) e trato alimentar e metabolismo com 19 medicamentos citados (Tabela 3). 21 (80,77\%) dos utentes utilizavam entre 1 e 2 medicamentos para o tratamento da asma. Quando questionados se os médicos prescritores orientavam sobre o uso correto dos medicamentos, $24(92,31 \%)$ responderam positivamente, e $21(80,77 \%)$ relataram que conseguem ler as receitas. 
Tabela 3 - Medicamentos utilizados pelos idosos.

\begin{tabular}{|c|c|c|c|}
\hline Grupo de medicamentos (ATC 1) & Grupo de medicamentos (ATC 2) & $\begin{array}{l}\text { Número de } \\
\text { Medicamentos }\end{array}$ & $\begin{array}{l}\text { Porcentagem em } \\
\text { relação ao total do } \\
\text { grupo ATC }\end{array}$ \\
\hline \multicolumn{4}{|l|}{ C: Sistema cardiovascular } \\
\hline & C09C Bloqueadores dos receptores da angiotensina II (BRA) & 16 & 26,6 \\
\hline & C10A Agentes modificadores lipídicos & 16 & 26,6 \\
\hline & $\begin{array}{l}\text { C08C Bloqueadores seletivos de canais de cálcio com principais efeitos } \\
\text { vasculares }\end{array}$ & 11 & 18,3 \\
\hline & C03A Diuréticos de ação moderada (Tiazidas) & 9 & 15 \\
\hline & C05C Agentes estabilizantes capilares & 3 & 5 \\
\hline & C07A Agentes Beta-bloqueadores & 2 & 3,4 \\
\hline & C01A Glicosídeos cardíacos & 1 & 1,7 \\
\hline & C01B Antiarrítmicos classe I e III & 1 & 1,7 \\
\hline & C01D Vasodilatadores utilizados em doenças cardíacas & 1 & 1,7 \\
\hline Total & & 60 & 100 \\
\hline \multicolumn{4}{|l|}{ R: Sistema respiratório } \\
\hline & $\begin{array}{l}\text { R03AK Adrenérgicos em combinação com corticosteroides ou outros } \\
\text { medicamentos }\end{array}$ & 26 & 47,3 \\
\hline & R03A Inalantes Adrenérgicos & 19 & 34,5 \\
\hline & $\begin{array}{l}\text { R03B Outros medicamentos para doenças obstrutivas das vias aéreas, } \\
\text { inalantes, Glicocorticoides }\end{array}$ & 6 & 10,9 \\
\hline & $\begin{array}{l}\text { R03D Outros medicamentos sistêmicos para doenças obstrutivas das } \\
\text { vias aéreas }\end{array}$ & 2 & 3,6 \\
\hline & R06 Anti-histamínicos de uso sistêmico & 1 & 1,8 \\
\hline & R01A Preparações nasais de uso tópico & 1 & 1,8 \\
\hline Total & & 55 & 100 \\
\hline \multicolumn{4}{|l|}{$\begin{array}{l}* A: \text { Trato alimentar e } \\
\text { metabolismo }\end{array}$} \\
\hline & $\begin{array}{l}\text { A02B Drogas para úlcera péptica e doença de refluxo gastresofágico } \\
\text { (DRGE) }\end{array}$ & 7 & 36,8 \\
\hline & A11A multivitaminas, combinações & 4 & 21,0 \\
\hline & A10B Drogas reduzidas de glicose sanguínea, exceto insulinas. & 3 & 15,8 \\
\hline & A11C vitamina A e D, incluindo combinações dos dois. & 3 & 15,8 \\
\hline & A03F Drogas para transtornos gastrointestinais funcionais & 1 & 5,3 \\
\hline & A12A suplementos minerais & 1 & 5,3 \\
\hline Total & & 19 & 100 \\
\hline \multicolumn{4}{|l|}{ N: Sistema nervoso } \\
\hline & N02B Outros analgésicos e antipiréticos & 4 & 66,7 \\
\hline & N06A Antidepressivos & 2 & 33,3 \\
\hline Total & & 6 & 100 \\
\hline \multicolumn{4}{|l|}{$\begin{array}{l}\text { M: Sistema músculo } \\
\text { esquelético }\end{array}$} \\
\hline & M01A Produtos anti-inflamatórios e antirreumáticos, não esteróides & 3 & 75 \\
\hline & M05B Medicamentos que afetam a estrutura óssea e a mineralização & 1 & 25 \\
\hline Total & & 4 & 100 \\
\hline \multicolumn{4}{|l|}{ S: Órgãos sensoriais } \\
\hline & S01E Preparações antiglaucoma e mióticos & 4 & 4 \\
\hline Total & & 4 & 100 \\
\hline \multicolumn{4}{|l|}{$\begin{array}{l}\text { B: Sangue e órgãos formadores } \\
\text { de sangue }\end{array}$} \\
\hline & B01A Agentes antitrombóticos & 2 & 100 \\
\hline Total & & 2 & 100 \\
\hline \multicolumn{4}{|l|}{$\begin{array}{l}\text { G: Sistema urinário gênito e } \\
\text { hormônios sexuais }\end{array}$} \\
\hline & G04B Urológicos & 2 & 100 \\
\hline Total & & 2 & 100 \\
\hline \multicolumn{4}{|l|}{$\begin{array}{l}\text { H: Preparações hormonais } \\
\text { sistêmicas, exceto hormônios } \\
\text { sexuais e insulinas }\end{array}$} \\
\hline & H03A Preparações para tireoide & 1 & 100 \\
\hline Total & & 1 & 100 \\
\hline \multicolumn{4}{|l|}{ V: Vários } \\
\hline & V03A Todos os outros produtos terapêuticos & 1 & 100 \\
\hline Total & & 1 & 100 \\
\hline
\end{tabular}

ATC- Anatomical Therapeutic Chemical. ATC 1 (primeiro nível de classificação por grupo anatômico), ATC 2-(segundo nível de classificação por grupo terapêutico). $\mathrm{n}=26$. Fonte: Cuidado farmacêutico em utentes idosos portadores de asma atendidos na farmácia ambulatorial do hospital universitário João de Barros Barreto, Belém, Pará. 
50\% dos idosos apresentaram mais de três diferentes problemas de saúde diagnosticados. Quando se observa dados do Brasil, a última pesquisa nacional de saúde apontou que aproximadamente $40 \%$ da população adulta, o equivalente a 57,4 milhões de pessoas, possui ao menos uma doença crônica não transmissível. A hipertensão arterial, o diabetes, a doença crônica de coluna, o colesterol e a depressão são as que apresentam maior prevalência no país, sendo que as duas primeiras têm aumento da prevalência com o passar da idade (Instituto Brasileiro de Geografia e Estatística, 2013). Além da asma a doença mais frequente nos idosos é a hipertensão arterial sistêmica com mais de $60 \%$, seguidas por dislipidemia $(42,3 \%)$, rinite $(15,40 \%)$, glaucoma $(11,50 \%)$ e diabetes $(11,50 \%)$. Nesse cenário, as associações encontradas reforçam a necessidade de um planejamento mais específico para a atenção a saúde aos idosos, incluindo os cuidados do profissional farmacêutico, justificado pela complexidade dos tratamentos farmacológicos (World Health Organization, 2015; 2020). O uso de múltiplos medicamentos acabam sendo comum nesta população devido a prevalência de doenças crônicas e às manifestações clínicas decorrentes do processo de envelhecimento (Tavares et al., 2015; Muniz et al., 2017; Nastaravičius \& Ramanauskienė, 2018; National Institutes of Health, 2018; Boulet et al., 2019).

\section{Problemas relacionados a medicamentos e intervenções farmacêuticas}

No decorrer dos seis meses de acompanhamento com os utentes identificou-se o total de 138 Problemas Relacionados a Medicamentos (PRMs). A Figura 3 apresenta a distribuição desses problemas por categorias, atestando os problemas de saúde insuficientemente tratados como o PRM mais prevalente (20,28\%), seguido por medicamentos administrados incorretamente (17,39\%), outros problemas de saúde que afetam o tratamento (15,21\%) e pela categoria outros PRMs com 13,04\%; ou seja, o principal PRM encontrado foi a identificação de problemas de saúde insuficientemente tratados. Estudos reconhecem que os dois PRMs mais prevalentes para essa população estão relacionados ao paciente apresentar um problema de saúde que requer terapia medicamentosa e o paciente ter um problema médico que é o resultado de não receber um medicamento (Sabzghabaee et al., 2012; Silva et al., 2013; Marchioro et al., 2014; Lemay et al., 2015; Peytremann-Bridevaux et al., 2015). 
Figura 3 - Distribuição de Problemas Relacionados a Medicamentos.

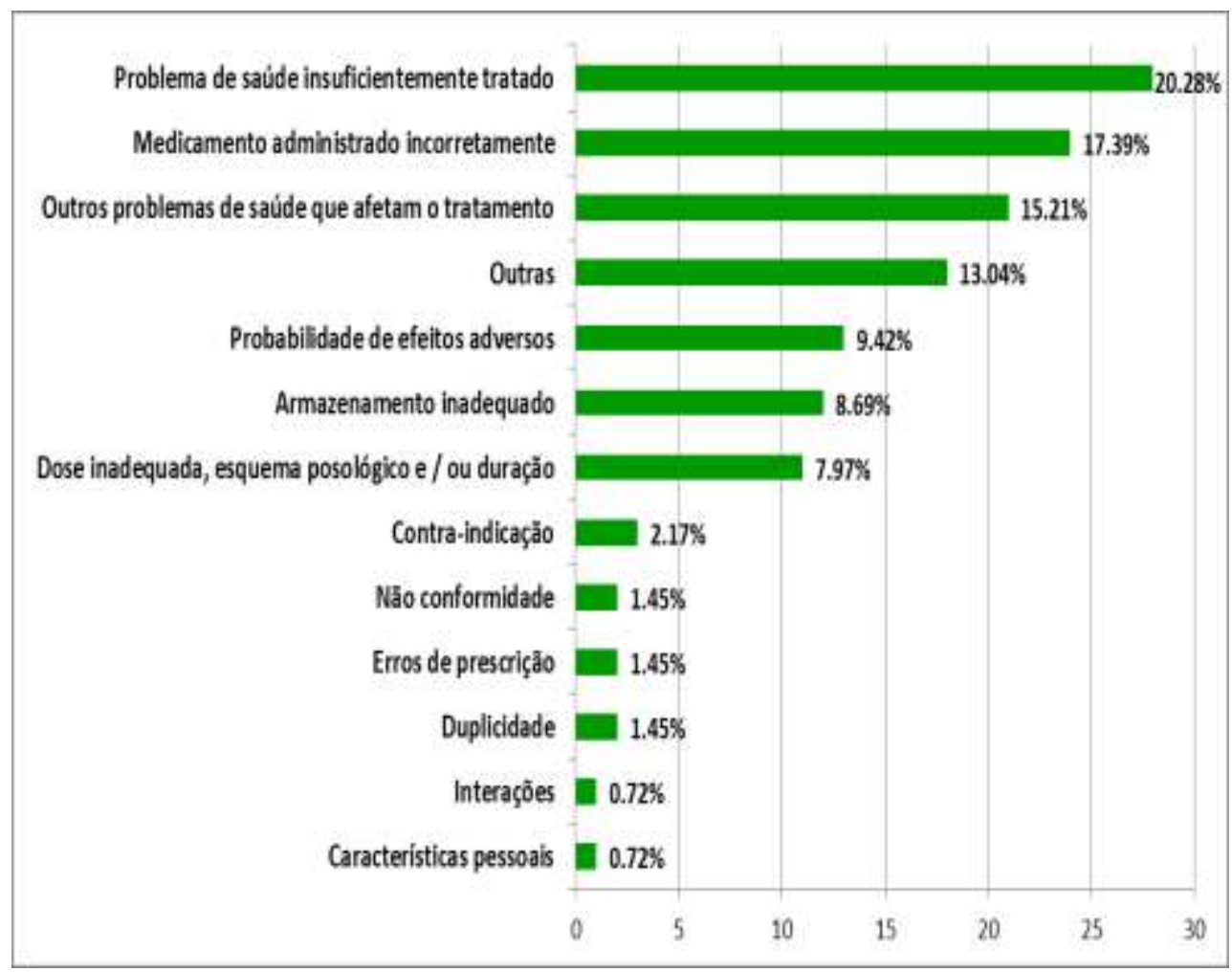

$\mathrm{n}=$ 26. Fonte: Cuidado farmacêutico em utentes idosos portadores de asma atendidos na farmácia ambulatorial do hospital universitário João de Barros Barreto, Belém, Pará.

A Figura 4 apresenta as intervenções farmacêuticas realizadas por categorias, chegando à totalidade de 750 intervenções executadas no período do acompanhamento farmacoterapêutico. Destacam-se as intervenções relacionadas à categoria Educação sobre a Asma, com 195 intervenções (24,53\% do total). Nesta categoria as intervenções mais frequentes foram o fornecimento de cartões com informações sobre o autocuidado relacionado asma (49), fornecimento de informações sobre a asma e seu tratamento (42), seguida do aconselhamento sobre a necessidade de reconhecer asma mal controlada e o que fazer caso houvesse piora do quadro (37).

Intervenções para fortalecer a adesão ao tratamento foi a segunda em quantidade realizadas com 171 ( $21,51 \%$ do total). As intervenções mais praticadas foram o fornecimento de tabelas com lembretes sobre o uso dos medicamentos (70), esclarecimentos sobre as possíveis consequências de não tomar os medicamentos (37) e ajustes de horários para a tomada dos medicamentos (27). Sobressaiu-se, também, as intervenções relacionadas ao uso do dispositivo inalatório 147 (18,47\% do total); onde a intervenção mais executada foi a revisão da técnica inalatória (80), seguida da demonstração correta da técnica inalatória com 66 intervenções. 
Figura 4 - Distribuição das intervenções farmacêuticas realizadas por categoria de intervenção

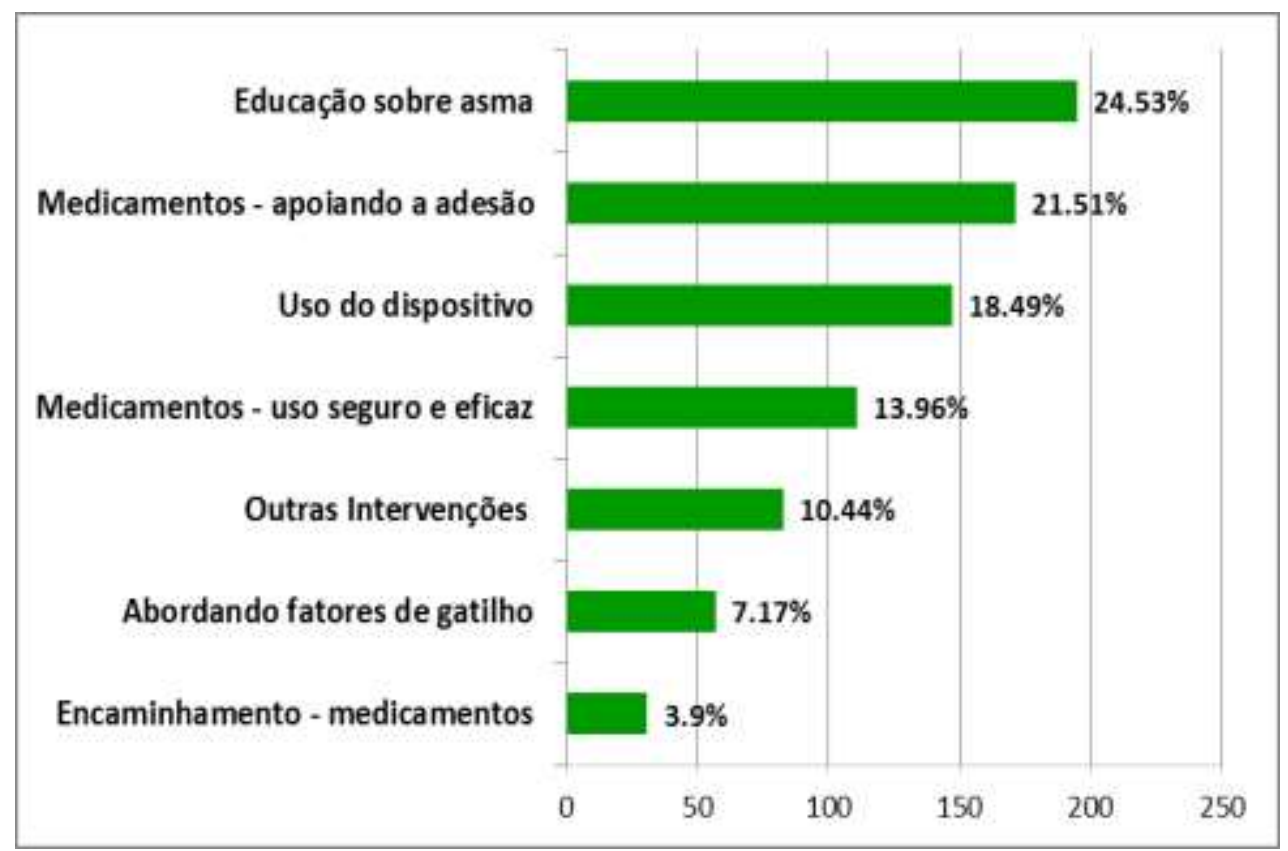

$\mathrm{n}=26$. Fonte: Cuidado farmacêutico em utentes idosos portadores de asma atendidos na farmácia ambulatorial do hospital universitário João de Barros Barreto, Belém, Pará.

Um estudo observacional envolvendo 340 utentes com idade superior a 60 anos, identificou como principal PRM nenhum medicamento prescrito, mas indicação clara da necessidade, evidenciando que havia problemas de saúde; no entanto, não havia oferta de tratamento (Hugtenburg, et al 2014). Outrossim, Silva et al., (2013) identificaram que os PRMs foram mais frequentes em utentes polimedicados com mais de 60 anos, onde mais de 50\% da amostra apresentava o PRM caracterizado como presença de um problema de saúde pela não utilização de um medicamento indicado.

Sabe-se, que para essa faixa etária, devido ao próprio processo natural do envelhecimento há uma maior predisposição para acometimentos por doenças crônicas, que geralmente são enfermidades complexas e bastante onerosas por necessitar de cuidados e exames contínuos e periódicos. Outrossim, há evidências de que pessoas que vivem em situação econômica mais precária estão mais expostas ao adoecimento e explica problemas de saúde insuficientemente tratados (Sabzghabaee et al., 2012; Silva et al., 2013; Marchioro et al., 2014; Lemay et al., 2015; Peytremann-Bridevaux et al., 2015).

Estudos identificando PRMs exclusivamente em utentes idosos com asma ainda são escassos, dificultando uma comparação mais precisa entre os achados. No entanto, já está bem documentado que na população com asma, os principais PRMs identificados estão relacionados com a administração dos medicamentos e aos esquemas terapêuticos inadequados (Amorha et al., 2018; Farias et al., 2016).

Dantas et al., (2019), em um estudo de acompanhamento farmacêutico em utentes ambulatoriais com asma, detectaram como PRM prevalente aquele relacionado ao cumprimento do regime terapêutico ou adesão ao tratamento, causado pelo não entendimento da técnica correta no uso dos medicamentos inalatórios (broncodilatadores e corticosteroides). Assim, inferem a urgente necessidade da incorporação de serviços farmacêuticos especializados em asma para minimizar os problemas encontrados e melhorar a qualidade de vida desse perfil de utentes.

Reafirma-se que o mapeamento dos PRMs foi importante para orientar quanto as intervenções a serem realizadas visando a resolução dos mesmos, de modo que, um foco sobre as informações, orientações e treinamentos sobre uso de medicamentos, sobretudo, dos dispositivos inalatórios foram priorizados no sentido de educar esses utentes a realizarem adequadamente o uso dos seus medicamentos. 
Crespo-Gonzalez et al., (2018) investigaram as principais categorias de intervenções fornecida por farmacêuticos no tratamento da asma, constatando que a maioria das intervenções foram direcionadas ao fornecimento de informações sobre medicamentos e o aconselhamento aos utentes, seguidas pela avaliação da técnica de inalação do paciente. Da mesma forma que, materiais educacionais e planos de ação escritos foram os materiais mais utilizados. Manfrin et al., (2017), ofereceram serviços farmacêuticos - estudo randomizado, controlado, multicêntrico e realizado na Itália - para utentes adultos com asma, utilizandose da revisão da farmacoterapia e de intervenções educacionais alcançando resultados clínicos significativos após as intervenções. Yadav e Thapa (2019) e Menegat et al., (2020), aplicaram técnicas educacionais utilizando folhetos, vídeos e tutoriais para demonstrar a técnica inalatória adequada alcançando excelentes desfechos. Dokbua et al., (2018), que estudaram os efeitos dos serviços de farmácia prestados por farmacêuticos comunitários para apoiar o autogerenciamento da asma - revisão sistemática com metanálise - conseguiram evidências significativas no controle da asma nos seguintes subgrupos: utentes com asma não controlada; intervenção de serviço composta de suporte a autogestão e provisão de plano de ação; duração curta do estudo de 6 meses ou menos; e ter um serviço adicional envolvendo a identificação e resolução de PRMs. Em adição, constatouse aumento significativo nas medidas de adesão a farmacoterapia (BMQ, p<0,01; MARS, p<0,01) e aumento significativo da qualidade de vida entre utentes com asma que receberam apoio de autogestão.

\section{Desfechos clínicos e humanísticos}

Na avaliação da adesão ao tratamento, no início do acompanhamento farmacoterapêutico, utilizando o Brief Medication Questionnaire (BMQ), identificou-se que 16 (61,53\%) dos idosos se encontravam com baixa adesão e 3 (11,54\%) com provável baixa adesão, enquanto 5 (19,23\%) foram classificados como tendo adesão e 2 (7,7\%) e provável adesão. Na avaliação final; ou seja ao final de seis meses, na última consulta farmacêutica, verificou-se que 12 (46,15\%) estavam na categoria de provável adesão e 5 (19,23\%) com adesão, 8 (30,77\%) com provável baixa adesão e 1 (3,85\%) com baixa adesão. A Figura 5 demonstra a representação gráfica com os valores dos escores obtidos na avalição inicial e final da adesão, evidenciando que houve diferença significativa $(\mathrm{p}<0,01)$ para adesão após seis meses de intervenção farmacêutica.

Figura 5 - Avaliação inicial e final de adesão a farmacoterapia.

\section{Avaliação da adesão a farmacoterapia (BMQ)}

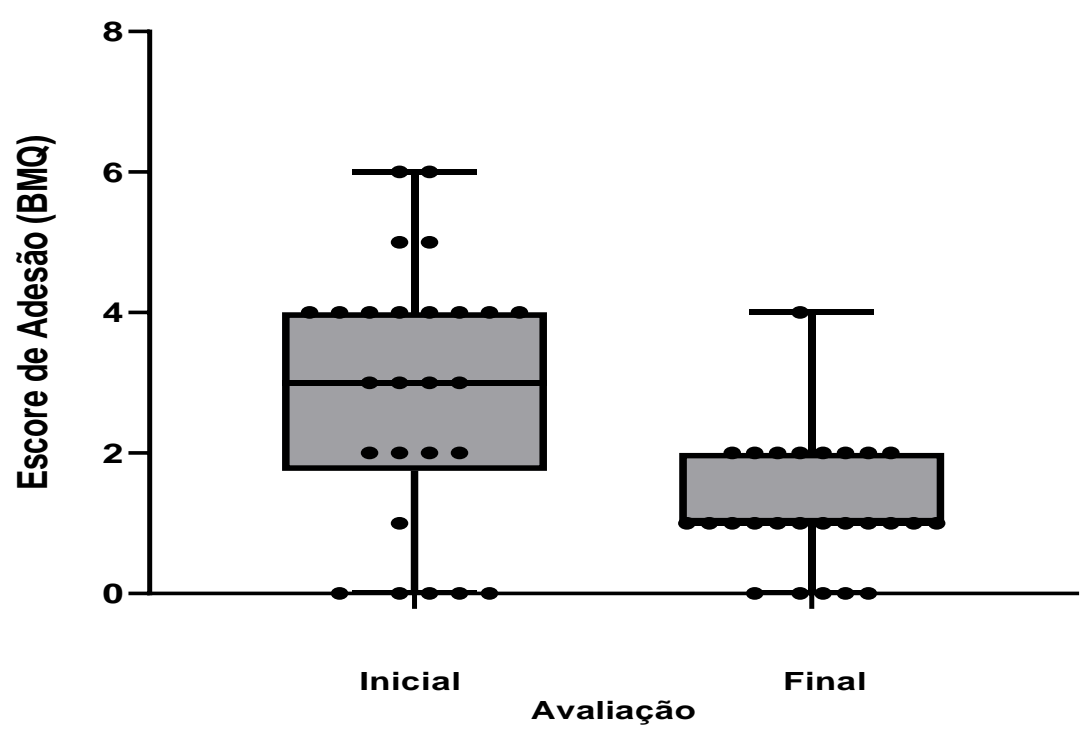

Box-plot com a distribuição escores após seis meses da oferta dos serviços farmacêuticos. Teste não-paramétrico de Wilcoxon, p < 0,01. Fonte: Cuidado farmacêutico em utentes idosos portadores de asma atendidos na farmácia ambulatorial do hospital universitário João de Barros Barreto, Belém, Pará. 
A Figura 6 demonstra o resultado da avaliação inicial e final do estado de controle da asma realizada por meio da pontuação no Avaliação do Controle da Asma (ACT). Observa-se que inicialmente que $16(57,69 \%)$ utentes apresentavam a asma parcialmente controlada e 7(30,77\%) asma descontrolada e 3 (11,54\%) com asma totalmente controlada. Na avaliação final, após seis meses de intervenção farmacêutica, foi achado que 16 (61,54\%) dos utentes estavam com a asma totalmente controlada, $10(38,46 \%)$ asma parcialmente controlada e 0,0 (0\%) como asma descontrolada.

Figura 6 - Avaliação inicial e final do controle da asma.

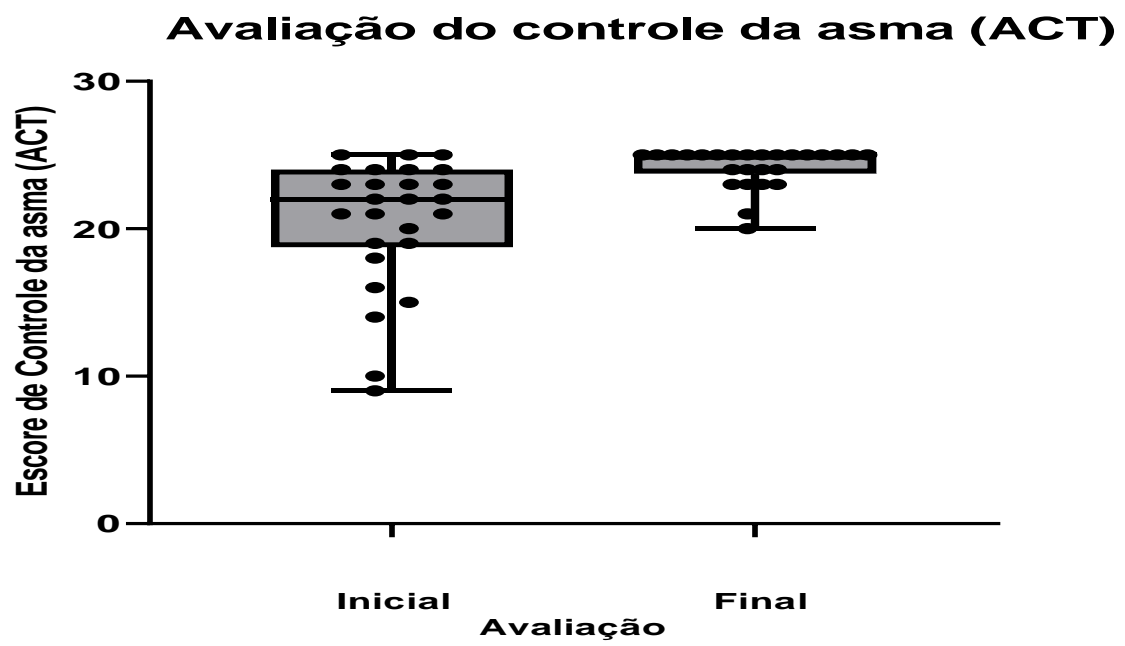

Box-plot com a distribuição escores após seis meses da oferta dos serviços farmacêuticos. Teste não-paramétrico de Wilcoxon, p < 0,01. Fonte: Cuidado farmacêutico em utentes idosos portadores de asma atendidos na farmácia ambulatorial do hospital universitário João de Barros Barreto, Belém, Pará.

Quanto a Avaliação do Fluxo Expiratório, observou-se uma diferença significativa (p < 0,01) quando comparados os valores de Pico de Fluxo inicial sem as intervenções e o Pico de Fluxo final, evidenciando aumento no fluxo expiratório após seis meses de intervenções farmacêuticas (Figura 7).

Figura 7 - Avaliação inicial e final do pico de fluxo expiratório

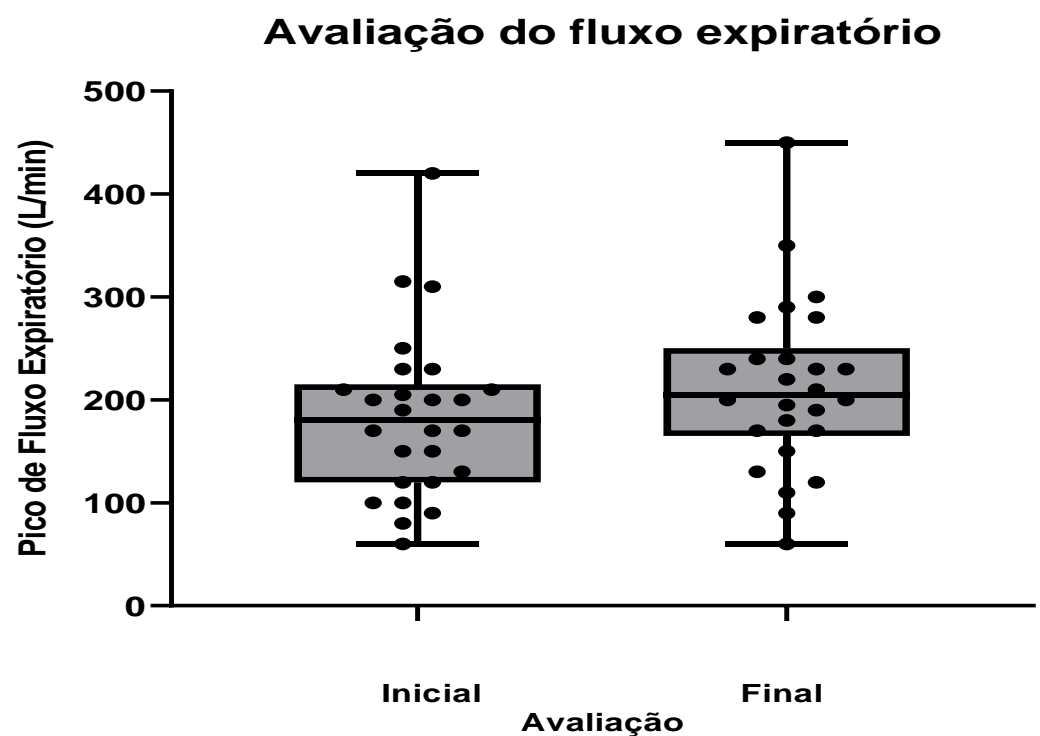

Box-plot com a distribuição escores após seis meses da oferta dos serviços farmacêuticos. Teste não-paramétrico de Wilcoxon, p<0,01. Fonte: Cuidado farmacêutico em utentes idosos portadores de asma atendidos na farmácia ambulatorial do hospital universitário João de Barros Barreto, Belém, Pará. 
A avaliação da Qualidade de Vida (QV) utilizando o instrumento World Health Organization Quality of Life Group Old (WHOQOL-OLD), identificou que no início do estudo 3 (11,54\%) utentes foram classificados com QV necessita melhorar, 17 (65,38\%) com QV regular e $6(23,08 \%)$ com QV boa. Na avaliação final, após as intervenções farmacêuticas, 0,0 (0\%) estava na categoria de $Q V$ necessita melhorar, 20 (76,92\%) QV boa e 6 (23,08\%) QV regular (Figura 8). Importante relatar que na avaliação inicial e na final houve utentes com QV muito boa; conforme pontuação do (WHOQOL-OLD).

Figura 8 - Avaliação inicial e final da qualidade de vida.

\section{Avaliação da qualidade de vida (WHOQOL-OLD)}

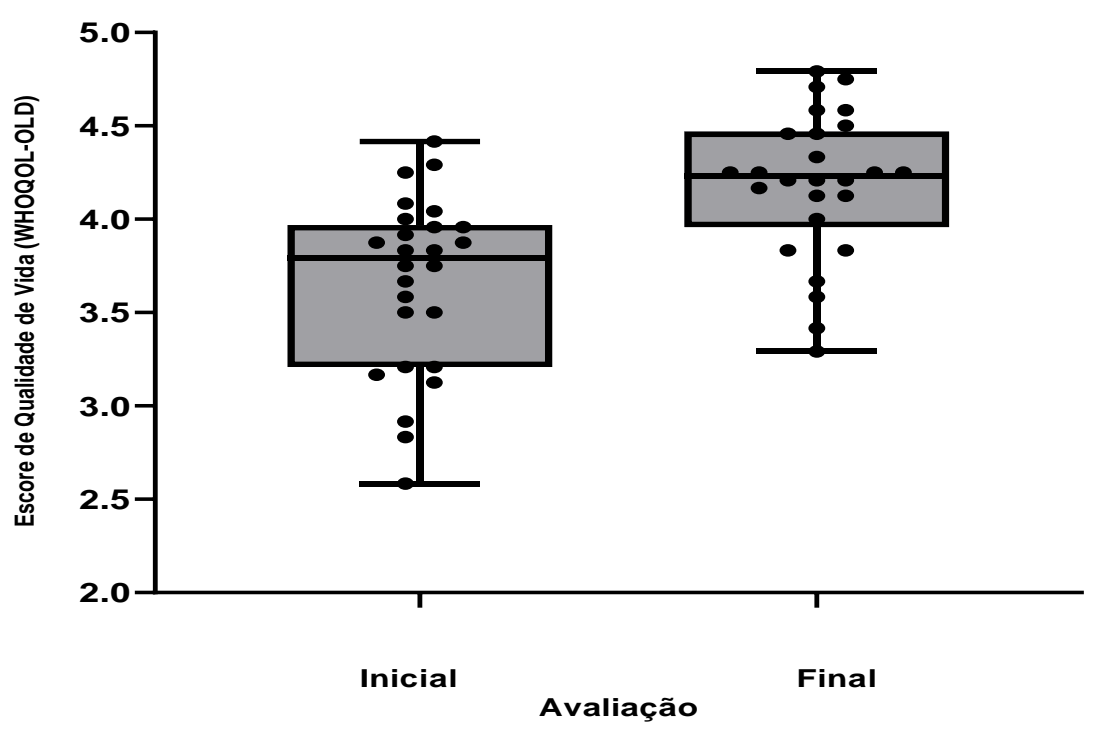

Box-plot com a distribuição escores após seis meses da oferta dos serviços farmacêuticos. Teste não-paramétrico de Wilcoxon, p<0,01. Fonte: Cuidado farmacêutico em utentes idosos portadores de asma atendidos na farmácia ambulatorial do hospital universitário João de Barros Barreto, Belém, Pará.

Reconhece-se que na asma, os diferentes dispositivos inalatórios e a execução equivocada da técnica, associadas à incompreensão de outros fatores que afetam o tratamento da doença acabam tornado difícil a adesão dos utentes ao tratamento (Anum et al., 2017; Kovacevic et al., 2018). Neste sentido, o cuidado farmacêutico tem um papel importante para melhorar a adesão ao tratamento e a qualidade de vida (Manfrin et al., 2017; Dokbua et al., 2018). Garcia-Cardenas et al., (2015), registram o impacto dos serviços farmacêuticos no controle da asma; ou seja, que os utentes que recebem cuidados farmacêuticos têm três vezes mais chances de ter sua asma controlada quando comparados aos que não recebem.

Peytremann-Bridevaux et al., (2015), apresentam evidências de que os programas de gerenciamento de doenças crônicas para adultos com asma podem melhorar a qualidade de vida, em especial para utentes asmáticos. Sabzghabaee et al., (2012), relatam os resultados de um estudo em um grupo de utentes asmáticos que receberam cuidados farmacêuticos; tais como educação em asma, aconselhamento sobre medicamentos, instruções sobre modificações no estilo de vida, diário de assistência à asma, entre outras, o desfecho final demonstra que as pontuações médias da Qualidade de Vida melhoraram significativamente após as intervenções farmacêuticas.

O farmacêutico - fundamentado em seu corpo esotérico do conhecimento -; ou seja, sobre a eficácia do fármaco, efetividade do medicamento e eficiência do tratamento, contribui para que o utente tenha uma participação mais ativa no processo terapêutico e na melhoria da sua qualidade de vida. 


\section{Contribuição, aplicações e avanços}

As ferramentas elaboradas e usadas no estudo foram incorporadas ao processo de trabalho; na rotina diária; assim como a implantação e implementação de Procedimentos Operacionais Padrão e/ou Guia para prestação do cuidado farmacêutico em utentes ambulatoriais com asma atendidos na farmácia ambulatorial do Hospital Universitário João de Barros Barreto da Universidade Federal do Pará.

\section{Limitação, viés e dificuldades}

Potenciais limitações podem estar relacionadas ao tamanho da amostra, recorte temporal do estudo e não ser multicêntrico. Potencial viés pode estar relacionado ao uso do WHOQOL-OLD, amplamente utilizado para avaliação da QV na população idosa, mas não desenvolvido especificamente para utentes idosos com asma. As dificuldades são aquelas inerentes as dificuldades de conciliar atividades de pesquisa e a rotina do processo de trabalho diário.

\section{Conflito de interesse}

Os autores declaram que a pesquisa foi conduzida na ausência de quaisquer relações comerciais ou financeiras que possam ser interpretados como um potencial conflito de interesse.

\section{Conclusão}

Os achados proporcionam um olhar sobre o perfil sociodemográfico, de morbidade e farmacoterapêutico, de adesão ao tratamento, de problemas relacionados a medicamentos, desfechos clínicos (avaliação do fluxo expiratório e avaliação do controle da asma), desfecho e humanístico (avaliação da qualidade de vida) e de intervenções farmacêuticas (organizacionais e educacionais) aplicados a utentes idosos com asma em tratamento para asma atendidos na Farmácia Ambulatorial do Hospital Universitário João de Barros Barreto (HUJBB) da Universidade Federal do Pará (UFPA).

Identificou-se necessidades, elaborou-se planos de cuidados individual, realizou-se diferentes intervenções que possibilitaram uma melhor adesão a farmacoterapia, controle da asma, fluxo expiratório e na qualidade de vida. Os resultados demonstram que as intervenções farmacêuticas - organizacionais e educacionais -, foram significativas para a melhoria da efetividade do tratamento e da qualidade de vida dos utentes. Estes achados são importantes para uma melhor compreensão do grupo de utentes idosos com asma - complexos -, com necessidades especificas, que precisam de manejo multidisciplinar; em especial o cuidado farmacêutico para a adequada prestação se serviços eficientes com qualidade.

Novas investigações são necessárias para aprimorar o conhecimento neste campo. Recomenda-se ampliar o estudo para o tipo caso-controle e econômico (custo-utilidade), visando aumentar a robustez dos resultados.

\section{Contribuições dos autores}

MWP concebeu o desenho do estudo sob orientação de ABAG e OS. MWP aplicou os instrumentos de pesquisa, analisou os resultados e escreveu o artigo sob a supervisão de ABAG e OS. Os autores leram e aprovaram a versão final do documento. O conteúdo do trabalho é de exclusiva responsabilidade dos autores.

\section{Referências}

Aguiar, R., Lopes, A., Ornelas, C., Ferreira, R., Caiado, J., Mendes, A., et al. (2017). Terapêutica inalatória: Técnicas de inalação e dispositivos inalatórios. Rev Port Imunoalergologia. 25(1):9-26.

Almomani, B. A., Mokhemer, E., Al-Sawalha, N. A., \& Momany, S. M. (2018). A novel approach of using educational pharmaceutical pictogram for improving inhaler techniques in patients with asthma. Respiratory medicine. 143, 103-108. https://doi.org/10.1016/j.rmed.2018.09.004 
Amegadzie, J. E., Gorgui, J., Acheampong, L., Gamble, J. M., Farrell, J., \& Gao, Z. (2021). Comparative safety and effectiveness of inhaled bronchodilators and corticosteroids for treating asthma-COPD overlap: a systematic review and meta-analysis. The Journal of asthma: official journal of the Association for the Care of Asthma. 58(3), 344-359. https://doi.org/10.1080/02770903.2019.1687716

Amorha, K. C., Onu, A. C., Anene-okeke, C. G., \& Ukwe, C. V. (2018). Evaluation of drug therapy problems in asthma patients receiving care in two hospitals in south-eastern nigeria. International Journal of Pharmacy and Pharmaceutical Sciences, 10(2), 50-55. https://doi.org/10.22159/ijpps.2018v10i2.22472

Andrade, T. N. G., Silvestre, C. C., Cunha, L. C., Silva, D. T., Marques, T. C., Oliveira-Filho, A. D., \& Lyra-Jr, D. P. (2015). Pharmaceutical intervention assessment in the identification and management of drug interactions in an intensive care unit. Journal of Applied Pharmaceutical Science. 5:13-8. 10.7324/JAPS.2015.50103

Anum, P. O., Anto, B. P., Forson, A. G. (2017). Structured pharmaceutical care improves the health-related quality of life of patients with asthma. $J$ Pharm Policy Pract. 10(8):1-9. http://doi.org/10.1186/s40545-017-0097-7

Ben, A. J., Neumann, C. R., \& Mengue, S. S. (2012). Teste de Morisky-Green e Brief Medication Questionnaire para avaliar adesão a medicamentos. Rev Saúde Pública. 46(2):279-89. 10.1590/S0034-89102012005000013

Brasil. (2014). Ministério da Saúde. Secretaria de Ciência, Tecnologia e Insumos Estratégicos. Departamento de Assistência Farmacêutica e Insumos Estratégicos. Capacitação para implantação dos serviços de clínica farmacêutica. Brasília: Ministério da Saúde. 308 p.: il. (Cuidado farmacêutico na atenção básica; caderno 2).

Brasil. Conselho Nacional de Saúde. (2012) Resolução nº 466 de 12 de dezembro de 2012. Aprova as diretrizes e normas regulamentadoras de pesquisas envolvendo seres humanos. Brasília. Distrito Federal. Ministério da Saúde (saude.gov.br)

Brasil. Conselho Nacional de Saúde. (2016). Resolução n ${ }^{\circ}$ 510, de 07 de abril de 2016. Dispõe sobre as normas aplicáveis a pesquisas em Ciências Humanas e Sociais cujos procedimentos metodológicos envolvam a utilização de dados diretamente obtidos com os participantes ou de informações identificáveis ou que possam acarretar riscos maiores o que os existentes na vida cotidiana. Brasília. Distrito Federal. Nova resolução (510/2016) de Ética na Pesquisa| ANPEd

Brasil. (2013). Ministério da Saúde. Protocolos Clínicos e Diretrizes Terapêuticas. 2. Segunda ed. 1-596p. Protocolos_Clinicos_e_Diretrizes_Terapeuticas_Volume_2.PDF (tre-rs.jus.br)

Brasil. (2014). Ministério da Saúde. Secretaria de Ciência, Tecnologia e Insumos Estratégicos. Departamento de Assistência Farmacêutica e Insumos Estratégicos. Serviços farmacêuticos na atenção básica à saúde. Brasília: Ministério da Saúde. 108p. il. - (Cuidado farmacêutico na atenção básica; Caderno 1). Cuidado farmacêutico na atenção básica. Caderno 1: Serviços farmacêuticos na atenção básica à saúde (saude.gov.br)

Cardoso, T. A., Roncada, C., Silva, E., Pinto, L. A., Jones, M. H., Stein, R. T., \& Pitrez, P. M. (2017). The impact of asthma in Brazil: a longitudinal analysis of data from a Brazilian national database system. Jornal brasileiro de pneumologia: publicação oficial da Sociedade Brasileira de Pneumologia e Tisilogia, 43(3):163-168. https://doi.org/10.1590/S1806-37562016000000352

Cavalcante, D., Oliveira, D., Antunes, M., \& Carnelozzi, A. (2018). Análise da qualidade de vida em idosas praticantes de dança. Revista Interdisciplinar de Promoção da Saúde. 1(1):23-31. 10.17058/rips.v1i1.11944

Chipps, B., Taylor, B., Bayer, V., Shaikh, A., Mosnaim, G., Trevor, J., et al. (2020). Relative efficacy, and safety of inhaled corticosteroids in asthma patients: systematic review and network meta-analysis. Ann Allergy, Asthma Immunol.;S1081-1206(20):30234-9. http://doi.org/10.1016/j.anai.2020.04.006

Costa, D. D., Pitrez, P. M., Barroso, N. F., \& Roncada, C. (2019). Asthma control in the quality of life levels of asthmatic patient's caregivers : a systematic review with meta-analysis and meta-regression. J Pediatr. 95(4):401-9. https://doi.org/10.1016/j.jped.2018.10.010

Crespo-Gonzalez, C., Fernandez-Llimos, F., Rotta, I., Correr, C. J., Benrimoj, S. I., \& Garcia-Cardenas, V. (2018). Characterization of pharmacists' interventions in asthma management: a systematic review. Journal Of The American Pharmacists Association. 58(2):210-219. 10.1016/j.japh.2017.12.009.

Dalcin, P. D. T. R., Grutcki, D. M., Laporte, P. P., Lima, P. B., Menegotto, S. M., \& Pereira, R. P. (2014). Fatores relacionados ao uso incorreto dos dispositivos inalatórios em utentes asmáticos. J Bras Pneumol. 40(1):13-20. https://doi.org/10.1590/S1806-37132014000100003

Dantas, S. C. C., Kheir, N., Dantas, F. F. L., Almeida, P. C. D., Silva, K. M., \& Fonteles, M. M. D. F. (2019). Pharmacotherapeutic follow-up of asthmatic patients in ambulatory care service of Ceará: clinical outcomes. Revista Brasileira de Farmácia Hospitalar e Serviços de Saúde. 3(3): 11-17. View of Pharmacotherapeutic follow-up of asthmatic patients in ambulatory care service of Ceará: clinical outcomes (rbfhss.org.br)

Dokbua, S., Dilokthornsakul, P., Chaiyakunapruk, N., Saini, B., Krass, I., \& Dhippayom, T. (2018). Effects of an Asthma Self-Management Support Service Provided by Community Pharmacists: a systematic review and meta-analysis. Journal Of Managed Care \& Specialty Pharmacy. 24(11):1184-1196. 10.18553/jmcp.2018.24.11.1184.

Farias, T. F., Aguiar, K. S., Rotta, I., Belletti, K. M. S., \& Carlotto, J. (2016). Implementing a clinical pharmacy service in hematology. Einstein (São Paulo). 14(3):384-390. 10.1590/s1679-45082016ao3667.

Ferreira, A. S., \& Soler, O. (2020). Strengthening patient safety strategies: an integrative review as to drug safety processes. Research, Society and Development, 9(12), e129129564. https://doi.org/10.33448/rsd-v9i12.9564

Flecka, M. P. A., Chachamovicha, E. \& Trentini, C. M. (2003). Projeto WHOQOL-OLD: método e resultados de grupos focais no Brasil. Rev Saúde Pública. 37(6):793-799. 16-AO3728WHOQOI.p65 (scielo.br)

Fuller, J. M., Saini, B., Bosnic-anticevich, S., Garcia, V., Benrimoj, S. I., \& Armour, C. (2017). Testing evidence routine practice : Using an implementation framework to embed a clinically proven asthma service in Australian community pharmacy. Res Soc Adm Pharm.;13(5):989-96. http://dx.doi.org/10.1016/j.sapharm.2017.05.019

Garcia-Cardenas, V., Armour, C., Benrimoj, S. I., Martinez-Martinez, F., Rotta, I., Fernandez-Llimos, F. (2015). Pharmacists' interventions on clinical asthma outcomes: a systematic review. European Respiratory Journal. 47(4):1134-1143. 10.1183/13993003.01497-2015. 
Global Initiative for Asthma. (2019). Global Strategy for Asthma Management and Prevention. https://ginasthma.org/gina-patient-guide-you-can-control-yourasthma

Ahmad, A., Mast, M. R., Nijpels, G., Elders, P. J., Dekker, J. M., \& Hugtenburg, J. G. (2014). Identification of drug-related problems of elderly patients discharged from hospital. Patient preference and adherence. 8:155-165. https://doi.org/10.2147/PPA.S48357

Instituto Brasileiro de Geografia e Estatística (IBGE). (2013). Pesquisa Nacional de Saúde: percepção do estado de saúde, estilos de vida e doenças crônicas Brasil, Grandes Regiões e Unidades da Federação Rio de Janeiro: IBGE; 2014.

Instituto Brasileiro de Geografia e Estatística (IBGE). (2019). Pesquisa de orçamentos familiares 2017-2018: primeiros resultados/Coordenação de Trabalho e Rendimento. IBGE. 69p.

Janežič, A., Locatelli, I., \& Kos, M. (2020). Inhalation technique and asthma outcomes with different corticosteroid-containing inhaler devices. The Journal of asthma : official journal of the Association for the Care of Asthma, 57(6):654-662. https://doi.org/10.1080/02770903.2019.1591442

Kovačević, M., Ćulafić, M., Jovanović, M., Vučićević, K., Kovačević, S. V., \& Miljković, B. (2018). Impact of community pharmacists' interventions on asthma self-management care. Research in social \& administrative pharmacy: RSAP. 14(6):603-611. https://doi.org/10.1016/j.sapharm.2017.07.007

Leite, M., Ponte, E. V., Petroni, J., Júnior, A. D. O., Pizzichini, E., \& Cruz, A. A. (2008). Avaliação do Questionário de Controle da Asma validado para uso no Brasil. J Bras Pneumol. 34(10):756-63. http://doi.org/10.1590/S1806-3713200800100000

Linneberg, A., \& Gonzalez-Quintela, A. (2016). The Unsolved Relationship of Alcohol and Asthma. International archives of allergy and immunology. 171(34)\;155-157. https://doi.org/10.1159/000454809

Lopes, I. C. C., Costa, J. L. B., Dias, J. B., Fialho, J. D. O., Pereira, B. C., \& Garib, J. R. (2013). Obesity, abdominal circumference and control of asthma symptomatology. Revista Médica de Minas Gerais. 23(1):21-26. 10.5935/2238-3182.20130004

Manfrin, A., Tinelli, M., Thomas, T., \& Krska, J. (2017). A cluster randomised control trial to evaluate the effectiveness and cost-effectiveness of the Italian medicines use review (I-MUR) for asthma patients. Bmc Health Services Research. 17(1):1-13. /10.1186/s12913-017-2245-9.

Marchioro, J., Gazzotti, M. R., Nascimento, O. A., Montealegre, F., Fish, J., \& Jardim, J. R. (2014). Level of asthma control and its relationship with medication use in asthma patients in Brazil. J Bras Pneumol. 40(5):487-494. 10.1590\%2FS1806-37132014000500004

Menegat, K. L., Borges, R. L., Senhorelo, M. C. C. M. P., \& Catro, P. F. S. (2020). Do health graduates know how to teach the technique of using inhalation devices? Brazilian J Dev. 6(4):19901-12. http://doi.org/10.34117/bjdv6n4-239

Minayo, M. C. S.; \& Costa, A. P. (2018). Fundamentos Teóricos das Técnicas de Investigação Qualitativa. Revista Lusófona de Educação, 40:139-153. ISSN 1645-7250

Muniz, E. C. S., Goulart, F. C., Lazarini, C. A., \& Marin, M. J. S. (2017). Analysis of medication use by elderly persons with supplemental health insurance zplans. Revista Brasileira de Geriatria e Gerontologia. 20(3):374-386. 10.1590/1981-22562017020.160111

Nastaravicius, A., \& Ramanauskiene, K. (2018). Role of a Community Pharmacy Service in Care of Bronchial Asthma Patients in Lithuania. Can Respir J. 2018:1-7. http://doi.org/10.1155/2018/6060581

Nastaravičius, A., \& Ramanauskienè, K. (2018). Role of a Community Pharmacy Service in Care of Bronchial Asthma Patients in Lithuania. Canadian Respiratory Journal. 2018:1-7. 10.1155/2018/6060581.

Nathan, R. A., Sorkness, C. A., Kosinski, M., Schatz, M., Li, J. T., Marcus, P., Murray, J. J., \& Pendergraft, T. B. (2004). Development of the asthma control test: a survey for assessing asthma control. J Allergy Clin Immunol. 113:59-65. 10.1016 / j.jaci.2003.09.008.

National Institutes Of Health. (2018). National Heart, Lung and Blood Institute. Global Initiative for Asthma: Global strategy for Asthma Management and Prevention. Washington: NIH. Global Initiative for Asthma - Global Initiative for Asthma - GINA (ginasthma.org)

Boulet, L. P., Reddel, H. K., Bateman, E., Pedersen, S., FitzGerald, J. M., \& O'Byrne, P. M. (2019). The Global Initiative for Asthma (GINA): 25 years later. The European respiratory journal. 54(2), 1900598. https://doi.org/10.1183/13993003.00598-2019

Oliveira, P. D., Menezes, A. M. B., Bertoldi, A. D., Wehrmeister, F. C., \& Macedo, S. E. C. (2014). Avaliação da técnica de utilização de dispositivos inalatórios no tratamento de doenças respiratórias no sul do Brasil: estudo de base populacional*. J Bras Pneumol. 40(5):513-20. http://doi.org/10.1590/S180637132014000500007

Oliveira, T. C., Vieira, H. K. S., Elmescany, S. B., Gonçalves, S. T., Santos, V. R. C., \& Soler, O. (2021). Interventions applied to the prescription, use and administration of medications as strategic factors for patient safety: systematic review. Research, Society and Development, 10(17), e195101724601. https://doi.org/10.33448/rsd-v10i17.24601

Paes, M. W., Soler, O., Grisólia, A. B.A. (2020). Intervenções farmacêuticas sobre o uso de dispositivos inalatórios: o cuidado farmacêutico no controle da asma. Infarma - Ciências Farmacêuticas. 32(4):309-318. ISSN - 2318-9312 (Versão eletrônica) 10.14450/2318-9312.v32.e4.a2020.pp309-318

Peytremann-Bridevaux, I., Arditi, C., Gex, G., Bridevaux, P. O., \& Burnand, B. (2015). Chronic disease management programmes for adults with asthma. The Cochrane Database of Systematic Reviews. 5:CD007988. 10.1002/14651858.cd007988.pub2.

Pizzichini, M. M. M., Carvalho-Pinto, R. M., \& Cançado, J. E. D. (2020). Recomendações para o manejo da asma da Sociedade Brasileira de Pneumologia e Tisiologia - 2020. J Bras Pneumol. 46(1): e201-307. 10.1590/1806-3713/e20190307

Power, M., Quinn, K., \& Schmidt, S. (2005). Development of the WHOQOL-Old Module. Quality of Life Research. 14(10):2197-2214. 10.1007/s11136-0057380-9 
Research, Society and Development, v. 11, n. 1, e59911125503, 2022

(CC BY 4.0) | ISSN 2525-3409 | DOI: http://dx.doi.org/10.33448/rsd-v11i1.25503

Price, D., Bosnic-Anticevich, J., Briggs, A., \& Chrystyn, H. (2013). The Inhaler Error Steering Committee, et al. Inhaler competence in asthma: common errors, barriers to use and recommended solutions. Respiratory Medicine. 107: 37-46. 10.1016/j.rmed.2012.09.017

Roxo, J. P. F., Ponte, E. V., Ramos, D. C. B., Pimentel, L., Júnior, A. D. O., \& Cruz, A. A. (2010). Validação do Teste de Controle da Asma em português para uso no Brasil. J Bras Pneumol. 36(2):159-66. http://doi.org/10.1590/S1806-37132010000200002

Sabzghabaee, A., Balasubramanian, R., Bhojan, C., Devarajan, V., Shanmugam, S., Varughese, J., Nair, M. S., \& Velu, S. (2012). Pharmaceutical care for asthma patients: a developing countrys experience. Journal Of Research In Pharmacy Practice. 1(2):66-78. 10.4103/2279-042x.108373.

Shanmugam, S., Varughese, J. N., Nair, M. A. S., Balasubramanian, R., Velu, S., Bhojan, C., et al. (2012). Pharmaceutical care for asthma patients: A Developing Country's Experience. J Res Phamacy Pract.1(2):66-71. http://doi.org/10.4103/2279-042X.108373

Silva, A. F., Abreu, C. R. O., Barbosa, E. M. S., Raposo, N. R. B., \& Chicourel, E. L. (2013). Problemas relacionados aos medicamentos em idosos fragilizados da Zona da Mata Mineira, Brasil. Revista Brasileira de Geriatria e Gerontologia. 16(4):691-704. 10.1590/s1809-98232013000400004.

Soler, O., \& Barreto, J. O. M. (2019). Community-Level pharmaceutical interventions to reduce the risks of polypharmacy in the elderly: Overview of systematic reviews and economic evaluations. Frontiers in Pharmacology. 10:Article 302.https://doi.org/10.3389/fphar.2019.00302

Tavares, N. U. L., Costa, K. S., Mengue, S. S., Vieira, M. L. F. P., Malta, D. C., \& Silva-Júnior, J. B. (2015). Uso de medicamentos para tratamento de doenças crônicas não transmissíveis no Brasil: resultados da pesquisa nacional de saúde, 2013. Epidemiologia e Serviços de Saúde. 24(2):315-323. 10.5123/s167949742015000200014

Thiollent, M. (2011). Metodologia da pesquisa-Ação. (18a ed.), Cortez.

Todo, B. A., \& Mota, P. A. (2009). Allergic respiratory diseases in the elderly. Respiratory Medicine. 103(11):1614-1622. 10.1016/j.rmed.2009.06.003. Word Health Organization (WHO). (2019). 10 facts on asthma 2019. Asthma (who.int)

World Health Organization (WHO). (2020). Chronic respiratory diseases: asthma. https://www.who.int/news-room/q-a-detail/asthma

World Health Organization (WHO). (2015). World report on ageing and health Geneva: World report on ageing and health (who.int)

Yadav, A., \& Thapa, P. (2019). Pharmacist Led Intervention on Inhalation Technique among Asthmatic Patients for Improving Quality of Life in a Private Hospital of Nepal. Pulm Med. (24):1-9. https://doi.org/10.1155/2019/8217901

Yin, R. K. (2015). Estudo de caso: planejamento e métodos. (5a ed.), Bookman. 320p.

Zambelli-Simões, L., Martins, M. C., Possari, J. C. C., Carvalho, G. B., Coelho, A. C. C., Cipriano, S. L. et al. (2015). Validação de escores de uso de dispositivos para inalação: valoração dos erros cometidos. Jornal Brasileiro de Pneumologia. 41(4):313-322. 10.1590/S1806-37132015000004435 